\title{
Index of Volume 21 (2001)
}

\section{Number 1}

$P$. Simon and F. Weisz

Hardy-Littlewood inequalities for two-parameter Vilenkin-Fourier coefficients $\ldots \ldots \ldots \ldots$. .

Y. Alber and S. Guerre-Delabriere

On the projection methods for fixed point problems $\ldots \ldots \ldots \ldots \ldots \ldots \ldots \ldots$

S. Feng

On Dirichlet type spaces, $\alpha$-Bloch spaces and $Q_{p}$ spaces on the unit ball of $C^{n} \ldots \ldots 41$

P. G. Todorov

Another look at the De Branges weight functions $\ldots \ldots \ldots \ldots \ldots \ldots \ldots \ldots \ldots$

D. Naylor

On an asymptotic expansion of the inverse Kontorovich-Lebedev transform 61

Ch. Pladdy

Asymptotics of the resolvent of the Dirac operator with a scalar short-range potential 79

C. Bénéteau and B. Korenblum

Jensen type inequalities and radial null sets

99

\section{Number 2}

Elijah Liflyand and Ferenc Móricz

The multi-parameter Hausdorff operator is bounded on the product Hardy space $H^{11}(\mathbb{R} \times \mathbb{R})$

Marianne Reichert

The weak semilinear Cauchy problem for ordinary differential equations 119

Mohammed A. Qazi

On polynomials monotonic on the unit interval 129 
Ilham Aliyev and Sasun Yakubov

Solution of irregular boundary value problems of ordinary differential equations

Friedrich Sauvigny

Deformation of boundary value problems for surfaces with proscribed

mean curvature

Yoshimi Saitō

Convergence of the Neumann Laplacian on shrinking domains

Karl-Heinz Indlekofer, Nikolai M. Timofeev

Multiplicative functions with bounded seminorm on the set of shifted primes

Dieter Gaier

On the decrease of Faber polynomials in domains with piecewise analytic boundary

\section{Number 3}

R. Picard, N. Weck and K. J. Witsch

Time-harmonic Maxwell equations in the exterior of perfectly conducting, irregular obstacles

W. Xiao and S. P. Zhou

The equivalence of Shepard operators in $\mathrm{L}^{\mathrm{p}}$ for $\mathrm{p} \geq 1$

J. Obermaier

The de la Vallée-Poussin kernel for orthogonal polynomial systems

S. Nevo

Application of Zalcman's lemma to $Q_{m}$-normal families 289 


\section{Number 4}

C. Elsner

Diophantine approximations with arithmetic restrictions and an application to trigonometric functions

E. Katsoprinakis, V. Nestoridis and I. Papadoperakis Universal Faber series

L. Lorch

Monotonicity and convexity of the sequences of the areas bounded by the sine and cosine integrals

O. Koch, P. Kofler and E. B. Weinmüller

Initial value problems for systems of ordinary first and second order differential

equations with a singularity of the first kind

M. Davidson

Partition polynomials and their zeros

B. Scarpellini

Stability by magnetic fields in the magnetic Bénard problem 403 



\section{Analysis}

\section{Volume 21 $\cdot$ Issue $4 \cdot 2001$}

C. Elsner

Diophantine approximations with arithmetic restrictions and an application to

trigonometric functions

E. Katsoprinakis, V. Nestoridis and I. Papadoperakis

Universal Faber series

L. Lorch

Monotonicity and convexity of the sequences of the areas bounded by the sine and cosine integrals

O. Koch, P. Kofler and E. B. Weinmüller

Initial value problems for systems of ordinary first and second order differential equations with a singularity of the first kind

M. Davidson

Partition polynomials and their zeros

B. Scarpellini

Stability by magnetic fields in the magnetic Bénard problem 403 
